\title{
Research on the Value and Innovation of Household Soft Outfit Exhibition Design Industry
}

\author{
Sun Wen \\ Guangdong University of Science \& Technology Department of Art and Design
}

Keywords: Household soft outfit; Exhibition design; Industrial value; Innovation research; Principles

\begin{abstract}
Many of the contemporary household decoration is stereotyped, and people are in desperate need of modern, personalized and unique household design to present the artistic charm and fashionable aesthetics of their houses. Household soft outfit has become a fashion for people's aesthetic philosophy at present. People re-decorate the furniture or ornamental that can be moved in their houses and design artistic works with unique styles and emotional appeals during household soft outfit. Therefore, social and scientific progress has improved people's life taste and quality intangibly. When high-grade things are designed in Chinese household soft outfit, people's aesthetic awareness is enhanced intangibly. Therefore, people have more confidence and energy to improve the quality of their interior decoration.
\end{abstract}

At present, the society develops more rapidly and people have had their unique artistic aesthetics and art enjoyment instead of solely surviving in the environment. As for household decoration, soft outfit exhibition design has become a mainstream for contemporary interior design. In the market, this soft outfit design is combined with hard outfit design; in the end, interior decoration with good conditions and artistic taste is designed, which is widely favored and loved by people. People believe that this design not only conforms to the comfortable environment requirement that they want, but also satisfies their aesthetic requirement of individual and elegant environment.

\section{Analysis of household soft outfit exhibition design industry}

Since people's life standard is rising rapidly, their aesthetic philosophy is also strengthening accordingly, their individual aesthetic taste is also becoming increasingly more enriched. In fact, household soft outfit exhibition design is an important link in household design. The traditional interior space design of "the same face in a thousand houses" has created aesthetic fatigue for people in the new era. Therefore, people's comprehensive yearning is to design a high-quality living environment with individual aesthetic taste.

It is widely known that household design is divided into soft design and hard design, and soft design means decoration activity that can be proceeded after hard decoration. It means second decoration of furniture that can be changed and updated after hard decoration. For example, changing furniture, lamps and so on and adding some ornamentals, such as artwork, paintings, treadmill and so on. Compared with hard decoration, soft decoration has a more comprehensive scope. Not only the interior environment and the economic conditions of the applicant should be taken into consideration, but also the environment atmosphere and the taste of the applicant should be considered. In the design process, from adjustment of environment and lamps, space with artistic characteristics can be created. According to requirements of the decoration applicant, the following furnishings can be established. Firstly, it is practical design, which means setting bed, sofa and other necessities in a certain location of the room. Secondly, it is interspersing design, which means interspersing some artwork, paintings and embroidery in it. Lastly, it is comprehensive design, which means placing things reasonably and paying attention to the coordination between surrounding environment and lighting.

The popularity of household soft outfit exhibition design industry is inseparable from its long history. It dated back from three thousand years ago. However, interior design at that time valued 
simple and plain mode. In later development, noble and magnificent mode appeared. With time going on, some religious elements were involved, and furniture like bed, tableware were brought in, and the colors were not restricted to white and blue. Gradually, under the influence of renaissance, the philosophy of soft outfit was changed to people-oriented philosophy, and the design content became increasingly more reasonable. With the continuous development of history, household soft outfit became more and more personalized, individual and fashionable, and the styles won more people's great favor. The previous exaggerated and monotonous characteristics were avoided. This design kept being updated, surprising and amazing people. In short, its historical development made it more reasonable and conformed to time more.

A designer should conform to the aesthetic philosophies, since only appreciators can promote its development. Therefore, household soft outfit exhibition design needs to obey the guidance of aesthetic design. Through the guidance of aesthetics, furniture, lamps and furnishings can be placed reasonably and matched harmoniously, thereby avoiding tedious or hollow visual effects. According to the harmony principle of aesthetics, all things in the house should be allocated reasonably, and color and styles should be matched. Aesthetic elements should be applied in it harmoniously. According to the high-lighting principle of aesthetics, things that one wants to highlight should be put among things in the room, and the highlighting thing can be set off. When they set off each other and match with each other, it will create visual impact. According to asymmetry principle in aesthetics, things in the room should be collocated with aesthetic principles, thereby getting rid of the traditional symmetric mode. However, everything should still be reasonable.

\section{Basic elements of household soft outfit exhibition design}

Household soft outfit exhibition design is an art form with loft aesthetic significance, and it has its own basic elements in the design process: art style, color language, texture application, architectural mass design and so on. The most important principle to apply these elements is to realize harmonious integration of them in a scientific and reasonable way.

When designers are designing, they must make sure of the style that they want to design, and good effects can only be achieved when the style matches with the goods. Soft outfit exhibition design involves its own personalized style; the artistic color of its design content is full-bodied, which suits the room very much. The design not only includes placing and arranging furniture, lamps and decoration in the room, but also color assortment of the surrounding environment as well as style matching of the series of things. For example, the application of traditional style, contemporary style and Western style. When designing household soft outfit exhibition design, the selection of style is very important, since it concerns whether it can reach the aesthetic philosophy of the decoration applicants and whether the designed household environment can reach personalized and comfortable requirements.

It is well known that color can create huge impacts to people's vision. Grasping color assortment well can cater to people's aesthetic philosophy directly and effectively. Once the colors are assorted well, it can trigger people's resonance directly and clearly, attract people's attention and create better effects. Therefore, if designers want to design good contents in household soft outfit exhibition design, they must match the colors well. Colors can be divided into two types, one is color with monochromatic light, and the other is color without monochromatic light. The former one is colorful, while the latter one is monotonous. These two different types of colors should be combined reasonably in different situations. For example, if one wants to create gorgeous and magnificent style, he should use colors with monochromatic light; while if one wants to create simple and plain style, he should use monotonous colors without monochromatic light. In fact, colors can be divided into warm tone and cool tone. Therefore, one should choose corresponding colors from warm tone and cool tone according to the style he wants to create. One can learn natural color collocation from nature, or learn the color assortment from brands by famous designers as well as refer to projects and plans by some famous designers.

In a house, different space has different functions, such as living room, master bedroom, kitchen, toilet and so on. Each space has different functions, and some corresponding adjustments in the 
layout need to be made to highlight corresponding functions. For example, living room is a major place for family members and guests to gather together. Therefore, openness needs to be obeyed in the arrangement of sofa, lamps and decoration, since it concerns the taste and favorite styles of family members. The master bedroom and secondary bedroom are major places for people to have a rest, so they should be closed and comfortable. These two points should be paid attention to in designing the layout, since it influences the success of the bedroom decoration. When warm tone is adopted in it, warm and comfortable feelings can be created, while cool tone might create repression for people. Some plants which can purify the air and feast the eyes can also be placed in advance. All in all, different atmosphere and styles should be created in different space. If one wants to present the characteristics and styles in a certain space, they must design corresponding content and present its special function.

\section{Research on the value and innovation of household soft outfit exhibition design industry}

With the continuous development of time, soft outfit exhibition design has become a contemporary trend in household decoration. Contemporary designers have been valuing soft outfit design and neglecting hard decoration. They pay attention to personalized, unique and fashionable design style. For example, traditional decoration style has disappeared in household decoration. Mixed style came into being, and lamps, embellishment and furniture are also included in the mixed style. Different styles begin to have reasonable collocation. In order to cater to the psychological philosophy of people's desire to get close to nature and environmentally friendly philosophy of social compulsive requirement, household soft outfit exhibition design is inclined to nature so that people can feel that they are in the nature and idyllic scenery. Household soft outfit exhibition design has improved people's life taste and quality, conformed to people's psychological and physical philosophies and enhanced the artistic colors of household, thereby triggering people's resonance in a larger degree, causing more sensation in the market and becoming contemporary trend.

Under the influence of market trend, many people begin to engage in household soft outfit exhibition design. Because of the market's desperate need of household soft outfit exhibition designers, it has brought development opportunities for soft outfit industry. Many people get rid of being habitual loafers, and the former habitual loafers begin to be devoted to soft outfit design industry. The continuous development has also proposed new requirements for soft outfit design, so this batch of people also need to improve their qualities and business abilities continuously, which endows soft outfit designers more power and purpose. With time going on, a batch of high-quality and capable talents are cultivated, which changed the condition of many habitual loafers or low-quality people in the country. From another perspective, it has promoted national development greatly and created communication ways for China and other countries. Therefore, Chinese culture is popularized and other countries can have more approaches to learn Chinese culture.

In order to avoid stagnant development of household soft outfit exhibition design, innovation must be created for new future. For example, the purpose of soft outfit industry is to serve for consumers. Therefore, in order to promote the development of household soft outfit exhibition design industry, consumers need to be guided appropriately to consume on household soft outfit. Consumers need to improve their own taste from soft outfit design, and corresponding people-oriented programs need to be constructed. Perfection is made from the essence to attract consumers and promote the development of soft outfit industry. In addition, in order to promote consumption, publicity needs to be enhanced in this industry. For example, a fully attractive website needs to be designed for forceful publicity, and some feedback can be publicized on it. With these two approaches, much attention can be drawn and consumers will be attracted to household soft outfit design in the end.

\section{Conclusions}

At present, soft outfit design has been very popular among modern people. However, it also has 
its own disadvantages. Therefore, it needs to keep going in development and keep innovation continuously. Personalized and reasonable design frameworks which conform more to people's aesthetic philosophies need to be designed. Only in this way can soft outfit design never be defeated and adapt to consumers' requirements more reasonably. Though domestic household soft outfit design started quite late, it has created a series of values; it has not only brought benefits for consumers, but also has cultivated numerous talents for the country.

\section{Acknowledgements}

Title: dongguan soft decoration design industry value and development trend research, is the Guangdong institute of science and technology, humanities and social sciences youth project, project number: gky - 2017kyqn - 21

\section{References}

[1] Liu Weihua, Audiences' Aesthetic Psychology and Artistic Pursuit of Exhibition Design [J], Chinese Museum, 2003(01)

[2] Zhou Chengmin, Xu Pan, On Decoration of Huayun—Chinese Style in Household Decoration [J], Furniture and Household Decoration, 2011(06)

[3] Zhao Ying, On Marginal Design that Can Determine Final Interior Design EffectsAccessory Art Design [J], Scientific News, 2010(15)

[4] Ma Qi. On Styles of Household Design [J], Kunming Metallurgy College, 2007(03)

[5] Peng Liang, Cathedral of Global Innovative Design and Fashionable Aesthetics_-A Report on 2011 Milan International Furniture Exhibition Design Trend [J], Furniture and Interior Design, 2011(06) 